\title{
On the Bott Cannibalistic Classes
}

\author{
By \\ Koichi HiRATA* and Akira Kono**
}

\section{§1. Introduction}

Let $G$ be a compact Lie group, $V$ a (complex) representation of $G$ and $\lambda_{V} \in K_{G}(V)$ the Thom class. Since $K_{G}(V)$ is a free $R(G)$-module generated by $\lambda_{V}$ (cf. [1]), there is an element $\theta_{k}(V) \in R(G)$ such that $\psi^{k}\left(\lambda_{V}\right)=\theta_{k}(V) \cdot \lambda_{V}$. The element $\theta_{k}(V)$ is called the Bott cannibalistic class of $V$.

By $K_{G}^{*}(\quad)$ we denote the $R O(G)$-graded equivariant $K$-theory. Since $\theta_{k}(V)$ is not a unit of $R(G)$, the Adams operation $\psi^{k}$ is not always a (stable) cohomology operation (cf. section 3). So the purpose of this paper is to show the following:

For a finite group $G, \psi^{k}$ is a cohomology operation of $K_{G}^{*}(\quad) \otimes Z\left[\frac{1}{k}\right]$ if and only if $(|G|, k)=1$ (for details see Theorem 3.1).

This paper is organized as follows: In section 2 we show that the element $\theta_{k}(V)$ is a unit of $R(G) \otimes Z\left[\frac{1}{k^{-}}\right]$if $(|G|, k)=1$. In the next section the main theorem is proved.

If $G$ is a $p$-group, then Atiyah and Tall showed that $\theta_{k}(V)$ is a unit of $R(G)$ $\otimes Z_{p}^{\hat{p}}$ (cf. [2]).

\section{§2. The Bott Camnibalistic Classes}

First recall the following properties of $\theta_{k}(V)$ (see [2]):

Lemma 2.1. Let $V$ and $W$ be representations of $G$ and $\varepsilon: R(G) \rightarrow Z$ the augmentation. Then

(i) $\theta_{k}(V+W)=\theta_{k}(V) \theta_{k}(W)$,

(ii) $\theta_{k}(V)=1+V+\cdots+V^{k-1}$ if $\operatorname{dim} V=1$,

(iii) $\varepsilon\left(\theta_{k}(V)\right)=k^{n}$ if $\operatorname{dim} V=n$.

Communicated by N. Shimada, March 12, 1982.

* Research Institute for Mathematical Sciences, Kyoto University, Kyoto 606, Japan.

** Department of Mathematics, Kyoto University, Kyoto 606, Japan. 
From now on we assume that $G$ is a finite group, $N, k$ integers such that $(N, k)=1$ and $|G|$ devides $N$.

Proposition 2.2. Let $V$ be a one dimensional representation of $G$. Then there exists a polynomial $f_{N, k}(x) \in Z\left[\frac{1}{k}\right][x]$ such that

$$
\theta_{k}(V) f_{N, h}(V)=1
$$

in $R(G) \otimes Z\left[\frac{1}{k}\right]$.

To prove Proposition 2.2 we need the following lemma:

Lemma 2.3. Let $R$ be a (commutative) ring (with unity) such that $k$ is invertible and $r \in R$. If $r^{N}=1$, then there exists $f_{N, k}(x) \in Z\left[\frac{1}{k}\right][x]$ such that

$$
\left(1+r+\cdots+r^{k-1}\right) f_{N, k}(r)=1 \text {. }
$$

Proof of Proposition 2.2. Since $V^{N}=1$ and $\theta_{k}(V)=1+V+\cdots+V^{k-1}$ by Lemma 2.1, $\theta_{k}(V) f_{N, k}(V)=1$ by Lemma 2.3.

Proof of Lemma 2.3. Let $\zeta$ be a primitive $N$-th root of 1 then we have

(i) $1+\zeta^{i}+\cdots+\zeta^{(N-1) i}=0$ for $1 \leqq i \leqq N-1$

and

(ii) $\prod_{i=1}^{N-1}\left(1-\zeta^{i}\right)=\prod_{i=1}^{N-1}\left(1-\zeta^{t i}\right) \neq 0 \quad$ if $\quad(t, N)=1$.

If we denote the matrix

$$
\left(\begin{array}{cccc}
x_{0} & x_{1} & \cdots & x_{N-1} \\
x_{N-1} & x_{0} & \cdots & x_{N-2} \\
& \cdots \cdots & \\
x_{1} & x_{2} & \cdots & x_{0}
\end{array}\right)
$$

by $A\left(x_{0}, x_{1}, \cdots, x_{N-1}\right)$ then

$$
\operatorname{det} A\left(x_{0}, x_{1}, \cdots, x_{N-1}\right)=\prod_{i=0}^{N-1}\left(x_{0}+\zeta^{i} x_{1}+\cdots+\zeta^{i(N-1)} x_{N-1}\right) .
$$

Since $(k, N)=1$, we can write $k=s N+t(s, t \in Z)$ with $1 \leqq t \leqq N-1$ and $(t, N)=1$. Put $u_{i}=s+1$ if $0 \leqq i<t$ and $u_{i}=s$ if $t \leqq i \leqq N-1$. Then

$$
1+r+\cdots+r^{k-1}=u_{0}+u_{1} r+\cdots+u_{N-1} r^{N-1}
$$

and 


$$
\begin{aligned}
\operatorname{det} & A\left(u_{0}, u_{1}, \cdots, u_{N-1}\right)=\prod_{i=0}^{N-1}\left(u_{0}+\zeta^{i} u_{1}+\cdots+\zeta^{i(N-1)} u_{N-1}\right) \\
= & k \prod_{i=1}^{N-1}\left(1+\zeta^{i}+\cdots+\zeta^{i(t-1)}+s\left(1+\zeta^{i}+\cdots+\zeta^{i(N-1)}\right)\right) \\
= & k \prod_{i=1}^{N-1}\left(1+\zeta^{i}+\cdots+\zeta^{i(t-1)}\right)=k\left(\left(\prod_{1=1}^{N-1}\left(1-\zeta^{i i}\right)\right) /\left(\prod_{i=1}^{N-1}\left(1-\zeta^{i}\right)\right)\right)=k .
\end{aligned}
$$

Since the determinant is a unit of $Z\left[\frac{1}{k}\right]$, there exists $a_{i} \in Z\left[\frac{1}{k}\right]$ such that

$$
\left(a_{0}, a_{1}, \cdots, a_{N-1}\right) A\left(u_{0}, u_{1}, \cdots, u_{N-1}\right)=(1,0, \cdots, 0) .
$$

Putting $f_{N, k}(x)=a_{0}+a_{1} x+\cdots+a_{N-1} x^{N-1}$, we have

$$
\left(1+r+\cdots+r^{k-1}\right) f_{N, k}(r)=1 \text {. }
$$

Define $h_{N, k}^{(n)}\left(X_{1}, \cdots, X_{n}\right) \in Z\left[\frac{1}{k}\right]\left[X_{1}, \cdots, X_{n}\right]$ by

$$
h_{N, k}^{(n)}\left(\sigma_{1}\left(x_{1}, \cdots, x_{n}\right), \cdots, \sigma_{n}\left(x_{1}, \cdots, x_{n}\right)\right)=\prod_{i=1}^{n} f_{N, k}\left(x_{i}\right),
$$

where $\sigma_{i}$ is the $i$-th elementary symmetric function. Then we have

Theorem 2.4. Let $V$ be an n-dimensional representation of $G$ and $k$ an integer such that $(|G|, k)=1$. Then

$$
\theta_{k}(V) h_{N, k}^{(n)}\left(\Lambda^{1}(V), \cdots, \Lambda^{n}(V)\right)=1
$$

in $R(G) \otimes Z\left[\frac{1}{k}\right]$.

Proof. Let $\left\{C_{\alpha}\right\}$ be all cyclic subgroups of $G$, then the restriction

$$
R(G) \longrightarrow \underset{\alpha}{\oplus} R\left(C_{\alpha}\right)
$$

is a monomorphism. Since $\theta_{k}, f_{N, k}$ and $h_{N, k}^{(n)}$ commute with restrictions, we may assume that $G$ is cyclic. Since every irreducible representation of a cyclic group is one dimensional, $V$ is a sum of one dimensional representations: $V=V_{1}$ $+V_{2}+\cdots+V_{n}$. Note that

$$
\Lambda^{i}(V)=\sigma_{i}\left(V_{1}, V_{2}, \cdots, V_{n}\right)
$$

and $\theta_{k}(V)=\prod_{i=1}^{n} \theta_{k}\left(V_{i}\right)$. Then Theorem 2.4 is an easy consequence of Proposition 2.2 .

Q.E.D.

Corollary 2.5. The element $\theta_{k}(V)$ is a unit of $R(G) \otimes Z\left[\frac{1}{k}\right]$ if $(|G|, k)=1$. 


\section{§3. Proof of the Main Theorem}

If $(|G|, k)=1$, then we can extend the Adams operation $\psi^{k}$ over $K_{G}^{*}(\quad)\left[\frac{1}{k}\right]$ by the commutative diagram

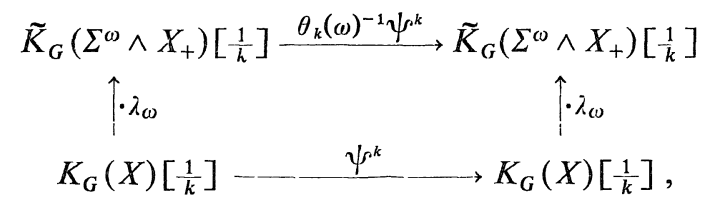

where $\omega$ is the regular representation of $G$.

Let $H$ be a subgroup of $G$ and $\operatorname{Ind}_{H}^{G}: R(H) \rightarrow R(G)$ the induction homomorphism. Recall that $K_{G}(G / H)=R(H)$ and $K_{G}(G / G)=R(G)$. Following Nishida [5], $\operatorname{Ind}_{H}^{G}$ is the transfer

$$
p_{*}: K_{G}(G / H) \longrightarrow K_{G}(G / G)
$$

for $p: G / H \rightarrow G / G$ and every transfer commutes with cohomology operations. So if $\psi^{k}: K_{G}^{*}(\quad)\left[\frac{1}{k}\right] \rightarrow K_{G}^{*}(\quad)\left[\frac{1}{k}\right]$ is a cohomology operation, $\operatorname{Ind}_{H}^{G} \circ \psi^{k}$ $=\psi^{k} \operatorname{Ind}_{H}^{G}: R(H)\left[\frac{1}{k}\right] \rightarrow R(G)\left[\frac{1}{k}\right]$. Moreover since $R(G)$ is torsion free, $\operatorname{Ind}_{H}^{G} \circ \psi^{k}=\psi^{k} \circ \operatorname{Ind}_{H}^{G}: R(H) \rightarrow R(G)$ if $\psi^{k}$ is a cohomology operation of $K_{G}^{*}(\quad)\left[\frac{1}{k}\right]-$ theory.

Now we can prove the following:

Theorem 3.1. Let $G$ be a finite group and $k$ an integer. Then the followings are equivalent:

(i) $(|G|, k)=1$,

(ii) for any representation $V$ of $G, \theta_{k}(V)$ is a unit of $R(G)\left[\frac{1}{k}\right]$,

(iii) $\psi^{k}: K_{G}^{*}(\quad)\left[\frac{1}{k}\right] \rightarrow K_{G}^{*}(\quad)\left[\frac{1}{k}\right]$ is a cohomology operation and

(iv) $\operatorname{Ind}_{H}^{G} \circ \psi^{k}=\psi^{k} \circ \operatorname{Ind}_{H}^{G}: R(H) \rightarrow R(G)$ for any subgroup $H$ of $G$.

Proof. Clearly it remains to prove that (iv) implies (i). Suppose (iv) is true and a prime $p$ devides $(|G|, k)$. Then there exists $g_{1} \in G$ such that $g_{1}$ is of order $p$ (cf. [3]). Put $H=\{1\}$, then $\operatorname{Ind}_{H}^{G}(1)=\omega$. Note that

$$
\chi_{\omega}(g)=\left\{\begin{array}{cl}
|G| & \text { if } g=1 \\
0 & \text { otherwise }
\end{array}\right.
$$

where $g \in G$ and $\chi_{\omega}$ is the character of the regular representation $\omega$ of $G$ (cf. [6]). Now we have 


$$
\chi_{\operatorname{Ind}_{H^{\circ}}^{G^{\circ} \psi^{h}(1)}}\left(g_{1}\right)=\chi_{\operatorname{Ind}_{H}^{G}(1)}\left(g_{1}\right)=\chi_{\omega}\left(g_{1}\right)=0
$$

and

$$
\chi_{\psi^{k}{ }^{k} \operatorname{Ind}_{H}^{G}(1)}^{G}\left(g_{1}\right)=\chi_{\psi^{k}(\omega)}\left(g_{1}\right)=\chi_{\omega}\left(g_{1}^{k}\right)=\chi_{\omega}(1)=|G| .
$$

This shows that $\operatorname{Ind}_{H}^{G} \circ \psi^{k} \neq \psi^{k} \operatorname{Ind}_{H}^{G}$, which contradicts to (iv).

Q.E.D.

\section{References}

[1] Atiyah, M. F., Bott periodicity and the index of elliptic operators, Quart. J. Math. Oxford (2), 19 (1968), 113-140.

[2] Atiyah, M. F. and Tall, D. O., Group representations, $\lambda$-rings and the $J$-homomorphism, Topology, 8 (1969), 253-297.

[ 3 ] Hall, M., The theory of groups, Macmillan, 1959.

[4] Kono, A., Induced representations of compact Lie groups and the Adams operations, Publ. RIMS, Kyoto Univ., 17 (1981), 553-556.

[5] Nishida, G., The transfer homomorphism in equivariant generalized cohomology theories, J. Math. Kyoto Univ., 18 (1978), 435-451.

[6] Serre, J-P., Représentation linéaires des groupes finis, Hermann, 1971. 
